\title{
An iterative, fast-sweeping-based eikonal solver for 3D tilted anisotropic media
}

\author{
Umair Bin Waheed ${ }^{1}$, Can Evren Yarman ${ }^{2}$, and Garret Flagg ${ }^{3}$
}

\begin{abstract}
Computation of first-arrival traveltimes for quasi-P waves in the presence of anisotropy is important for high-end nearsurface modeling, microseismic-source localization, and fractured-reservoir characterization - and it requires solving an anisotropic eikonal equation. Anisotropy deviating from elliptical anisotropy introduces higher order nonlinearity into the eikonal equation, which makes solving the eikonal equation a challenge. We addressed this challenge by iteratively solving a sequence of simpler tilted elliptically anisotropic eikonal equations. At each iteration, the source function was updated to capture the effects of the higher order nonlinear terms. We used Aitken's extrapolation to speed up convergence rate of the iterative algorithm. The result is an algorithm for computing first-arrival traveltimes in tilted anisotropic media. We evaluated the applicability and usefulness of our method on tilted transversely isotropic media and tilted orthorhombic media. Our numerical tests determined that the proposed method matches the first arrivals obtained by wavefield extrapolation, even for strongly anisotropic and highly complex subsurface structures. Thus, for the cases where two-point ray tracing fails, our method can be a potential substitute for computing traveltimes. The approach presented here can be easily extended to compute first-arrival traveltimes for anisotropic media with lower symmetries, such as monoclinic or even the triclinic media.
\end{abstract}

\section{INTRODUCTION}

Sedimentary rocks exhibit anisotropic wave propagation behavior due to the thin layering of transversely isotropic (TI) rocks with different properties (Stoep, 1966). If the layering is horizontal, this gives rise to a TI medium with a vertical axis of symmetry (VTI). However, tectonic movements of the crust and migration of salt bodies may cause tilt and rotation of the plane containing the symmetry axis, giving rise to a TI medium with a tilted axis of symmetry (TTI). Many sedimentary formations, including sands and carbonates, and natural fracture networks contain vertical or steeply dipping fracture sets and should be described by symmetries lower than TI, such as orthorhombic (Bakulin et al., 2000; Tsvankin et al., 2010). In the presence of tilt and rotation, the symmetry is referred to as tilted orthorhombic (TOR).

For high-end near-surface modeling, microseismic-source localization, and fractured-reservoir characterization, it is important to compute first-arrival traveltimes in the presence of anisotropy (Baofu et al., 2008; Taillandier et al., 2009; Al-Shuhail et al., 2013). First-arrival traveltimes satisfy the eikonal equation, which is obtained from the high-frequency asymptotic approximation of the wave equation. Ray tracing and finite difference methods are the two main approaches used to compute numerical solutions to the eikonal equation.

Ray tracing methods aim to compute traveltime along the characteristics, also referred to as rays, of the eikonal equation by solving a system of ordinary differential equations (Červený, 2001). Each ray corresponds to an individual elementary wave, such as first arrival, direct, reflected, multiply reflected, converted, etc. For practical applications, such as imaging and model building, the traveltimes computed along rays are required to be interpolated between the rays (Waheed et al., 2013). This computation is a challenge, especially in complex media, where the rays may diverge from each other, eventually leading to large spatial gaps between the rays. In light of these challenges, several approaches to solving the eikonal equation using finite differences (Vidale, 1990; van Trier and Symes, 1991; Sethian and Popovici, 1999; Qian and Symes, 2001) have been developed. One advantage of this approach

Manuscript received by the Editor 11 August 2014; revised manuscript received 11 November 2014; published online 30 March 2015.

${ }^{1}$ King Abdullah University of Science and Technology, Physical Sciences and Engineering Division, Makkah, Saudi Arabia. E-mail: umairbin.waheed@kaust .edu.sa.

${ }^{2}$ Schlumberger, Cambridge, UK. E-mail: cyarman@slb.com.

${ }^{3}$ Schlumberger, Houston, Texas, USA. E-mail: gflagg@slb.com.

(C) 2015 Society of Exploration Geophysicists. All rights reserved. 
is that the solutions always provide a traveltime between two points in a given model. Although limited to first-arrival traveltimes, these eikonal solvers can be extended to image multiple arrivals (Bevc, 1997). Initial attempts to incorporate seismic anisotropy into eikonal solvers can be traced back to Dellinger and van Trier (1990), Dellinger and Symes (1997), etc.

Finite-difference solutions do not treat individual elementary waves separately, but they solve for the first-arrival times corresponding to the complete wavefield. The different approaches proposed to obtain numerical solutions of the eikonal equation can be classified as (1) embedding methods, (2) iterative methods, (3) single-pass methods, and (4) sweeping methods. The embedding methods solve the eikonal equation by transforming it into a dynamic Hamilton-Jacobi equation (Osher, 1993), which entails a computationally costly procedure. Iterative methods begin with an initial traveltime distribution and use minimization techniques to update this initial guess (Rouy and Tourin, 1992). The approach can take a significantly long time to converge, especially in the presence of highly heterogeneous and/or strongly anisotropic media.

Single-pass methods were initially developed for isotropic eikonal equations (Sethian and Popovici, 1999). These methods rely on the fact that the direction of the energy conveyed along a wave, referred to as the group velocity, is perpendicular to the wavefront direction, which is computed by the traveltime gradient and referred to as the phase velocity. For anisotropic media, the traveltime gradient cannot be used as a reliable indicator of energy flow because the group velocity vector deviates from the phase velocity. Hence, such methods are no longer applicable in the presence of anisotropy. Sethian and Vladimirsky (2003) modify the algorithm to account for anisotropy; however, the computational load is increased proportional to the strength of anisotropy in the medium. Konukoglu et al. (2007) and Cristiani (2009) propose variations of the original single-pass method to account for anisotropic propagation. However, these methods deal with a simpler class of the anisotropic problem, the tilted elliptically anisotropic (TEA) case.

Similar to single-pass methods, the originally proposed fast-sweeping method (Zhao, 2005) deals with the isotropic case only. To handle the presence of anisotropy in the medium, modifications to the original method are proposed by Tsai et al. (2003), Zhang et al. (2006), and Qian et al. (2007) to solve the TEA eikonal equation. This iterative framework is more robust and flexible for general equations than the single-pass method. Additionally, it is more efficient, both computationally and in terms of memory requirement for production size models because we do not need to keep track of the wavefront, which can grow to be extremely large in such models.

Anisotropy deviating from elliptical anisotropy introduces higher order nonlinear terms into the eikonal equation, which makes it challenging to solve (Alkhalifah and Fomel, 2011; Waheed et al., 2015). This challenge can be addressed by iteratively solving a sequence of much simpler TEA eikonal equations (Stovas and Alkhalifah, 2012; Waheed and Alkhalifah, 2013). At each iteration, the source function is updated to capture the effects due to the higher order nonlinear terms. Ma and Alkhalifah (2013) use this approach to solve the 2D VTI eikonal equation.

In this paper, we use an iterative TEA-based method for computation of first-arrival traveltimes in TTI and TOR media (Waheed et al., 2014). We combine the fixed-point iteration approach with Aitken's extrapolation method (Atkinson, 1989) to accelerate convergence rate of the iterative scheme. The result is an algorithm for computing first- arrival traveltimes in tilted anisotropic media. At each iteration, we use the fast-sweeping method to solve a TEA eikonal equation. Our approach can also be extended to anisotropic media with lower symmetries, such as the monoclinic or even the triclinic media.

The rest of the paper is organized as follows: We begin with background information on the anisotropic eikonal equation. This is followed by description of the method used to solve the eikonal equation. Finally, we present numerical tests demonstrating the usefulness and applicability of the proposed algorithm to TTI (2D/3D) and TOR (3D) media.

\section{BACKGROUND}

\section{Tilted orthorhombic eikonal equation}

The stiffness coefficient matrix for an orthorhombic model can be represented using the compressed Voigt notation, as follows:

$$
\mathbf{C}=\left[\begin{array}{cccccc}
c_{11} & c_{12} & c_{13} & 0 & 0 & 0 \\
c_{21} & c_{22} & c_{23} & 0 & 0 & 0 \\
c_{31} & c_{32} & c_{33} & 0 & 0 & 0 \\
0 & 0 & 0 & c_{44} & 0 & 0 \\
0 & 0 & 0 & 0 & c_{55} & 0 \\
0 & 0 & 0 & 0 & 0 & c_{66}
\end{array}\right]
$$

Alternatively, one can use the parameterization introduced by Alkhalifah (2003):

$$
\begin{aligned}
& v_{0}=\sqrt{\frac{c_{33}}{\rho}, \quad v_{s 1}=\sqrt{\frac{c_{55}}{\rho}}, \quad v_{s 2}}=\sqrt{\frac{c_{44}}{\rho}}, \quad v_{s 3}=\sqrt{\frac{c_{66}}{\rho}}, \\
& v_{1}=\sqrt{\frac{c_{13}\left(c_{13}+2 c_{55}\right)+c_{33} c_{55}}{\rho\left(c_{33}-c_{55}\right)}}, \\
& v_{2}=\sqrt{\frac{c_{23}\left(c_{23}+2 c_{44}\right)+c_{33} c_{44}}{\rho\left(c_{33}-c_{44}\right)}}, \\
& \eta_{1}=\frac{1}{2 c_{13}\left(c_{13}+2 c_{55}\right)+2 c_{33} c_{55}}-\frac{1}{2}, \\
& \eta_{2}=\frac{c_{22}\left(c_{33}-c_{44}\right)}{2 c_{23}\left(c_{23}+2 c_{44}\right)+2 c_{33} c_{44}}-\frac{1}{2}, \\
& \delta=\frac{\left(c_{12}+c_{66}\right)^{2}-\left(c_{11}-c_{66}\right)^{2}}{2 c_{11}\left(c_{11}-c_{66}\right)},
\end{aligned}
$$

where $\rho$ is the density, $v_{0}$ is the quasi-P (qP) wave vertical velocity; $v_{s 1}$ and $v_{s 2}$ are the quasi-S (qS) wave vertical velocities polarized in the $[y, z]$ and $[x, z]$ planes, respectively; $v_{s 3}$ is the $\mathrm{qS}$ wave horizontal velocity polarized in the $[x, z]$ plane but propagating in the $x$-direction; $v_{1}$ and $v_{2}$ are the normal moveout (NMO) qP wave velocities for horizontal reflectors in the $[x, z]$ and $[y, z]$ planes, respectively, $\eta_{1}$ and $\eta_{2}$ are the anellipticity anisotropy parameters in the $[x, z]$ and $[y, z]$ planes, respectively; and the Thomsen's VTI parameter $\delta$ is defined in the $[x, y]$ plane (with respect to the $x$-coordinate axis).

It is demonstrated by Alkhalifah $(1998,2003)$ that for TI and orthorhombic media, setting the qS-wave velocity along the symmetry axis to zero does not compromise the accuracy of the qP-wave traveltime computations. Consequently, the eikonal equation for the qP-wave in an orthorhombic medium can be written as 


$$
\begin{aligned}
& A\left(\partial_{x} \tau\right)^{2}+B\left(\partial_{y} \tau\right)^{2}+C\left(\partial_{z} \tau\right)^{2}+D\left(\partial_{x} \tau\right)^{2}\left(\partial_{y} \tau\right)^{2} \\
& \quad+E\left(\partial_{x} \tau\right)^{2}\left(\partial_{z} \tau\right)^{2}+F\left(\partial_{y} \tau\right)^{2}\left(\partial_{z} \tau\right)^{2} \\
& \quad+G\left(\partial_{x} \tau\right)^{2}\left(\partial_{y} \tau\right)^{2}\left(\partial_{z} \tau\right)^{2}=1
\end{aligned}
$$

where $\tau(x, y, z)$ is the traveltime measured from the source to a point with coordinates of $(x, y, z)$ and $\partial_{x} \tau, \partial_{y} \tau, \partial_{z} \tau$ are the traveltime derivatives with respect to the $x$-, $y$-, and $z$-directions, respectively. The following definitions have been used for simplification:

$$
\begin{aligned}
A= & v_{1}^{2}\left(1+2 \eta_{1}\right), \quad B=v_{2}^{2}\left(1+2 \eta_{2}\right), \quad C=v_{0}^{2}, \\
D= & v_{1}^{2}\left(1+2 \eta_{1}\right)\left(\left(1+2 \eta_{1}\right) \gamma^{2} v_{1}^{2}-\left(1+2 \eta_{2}\right) v_{2}^{2}\right), \\
E= & -2 \eta_{1} v_{1}^{2} v_{0}^{2}, \quad F=-2 \eta_{2} v_{2}^{2} v_{0}^{2}, \\
G= & -v_{0}^{2} v_{1}^{2}\left(\left(1+2 \eta_{1}\right)^{2} v_{1}^{2} \gamma^{2}-2\left(1+2 \eta_{1}\right) v_{1} v_{2} \gamma\right. \\
& \left.+\left(1-4 \eta_{1} \eta_{2}\right) v_{2}^{2}\right) .
\end{aligned}
$$

The parameter $\gamma$ is defined in terms of the $\delta$ parameter through the relation $\gamma=\sqrt{1+2 \delta}$.

For the TOR medium, the traveltime derivatives in equation 3 are evaluated in the rotated coordinate frame:

$$
\begin{aligned}
\widehat{\partial_{x} \tau}= & (\cos \phi \cos \psi \cos \theta-\sin \phi \sin \psi)\left(\partial_{x} \tau\right) \\
& +(\cos \phi \sin \psi+\cos \psi \sin \phi \cos \theta)\left(\partial_{y} \tau\right) \\
& +(\cos \psi \sin \theta)\left(\partial_{z} \tau\right), \\
\widehat{\partial_{y} \tau}= & (-\cos \psi \sin \phi-\cos \phi \cos \theta \sin \psi)\left(\partial_{x} \tau\right) \\
& +(\cos \phi \cos \psi-\cos \theta \sin \phi \sin \psi)\left(\partial_{y} \tau\right) \\
& -(\sin \psi \sin \theta)\left(\partial_{z} \tau\right), \\
\widehat{\partial_{z} \tau}= & -(\cos \phi \sin \theta)\left(\partial_{x} \tau\right)-(\sin \phi \sin \theta)\left(\partial_{y} \tau\right) \\
& +(\cos \theta)\left(\partial_{z} \tau\right),
\end{aligned}
$$

where $\theta$ is the dip angle, $\phi$ denotes the azimuthal angle, and $\psi$ is the rotation angle. Then, the corresponding eikonal equation for TOR media is given by

$$
\begin{aligned}
& A\left(\widehat{\partial_{x} \tau}\right)^{2}+B\left(\widehat{\partial_{y} \tau}\right)^{2}+C\left(\widehat{\partial_{z} \tau}\right)^{2}+D\left(\widehat{\partial_{x} \tau}\right)^{2}\left(\widehat{\partial_{y} \tau}\right)^{2} \\
& +E\left(\widehat{\partial_{x} \tau}\right)^{2}\left(\widehat{\partial_{z} \tau}\right)^{2}+F\left(\widehat{\partial_{y} \tau}\right)^{2}\left(\widehat{\partial_{z} \tau}\right)^{2}+G\left(\widehat{\partial_{x} \tau}\right)^{2}\left(\widehat{\partial_{y} \tau}\right)^{2}\left(\widehat{\partial_{z} \tau}\right)^{2}=1
\end{aligned}
$$

\section{The 3D tilted axis of symmetry eikonal equation}

For

$$
v_{1}=v_{2}=v_{\mathrm{nmo}}, \quad \eta_{1}=\eta_{2}=\eta, \quad \gamma=1,
$$

equation 6 reduces to the eikonal equation for a TTI medium (Stovas and Alkhalifah, 2012):

$$
\begin{aligned}
& v_{\mathrm{nmo}}^{2}(1+2 \eta)\left(\left(\widehat{\partial_{x} \tau}\right)^{2}+\left(\widehat{\partial_{y} \tau}\right)^{2}\right) \\
& \quad+v_{0}^{2}\left(\widehat{\partial_{z} \tau}\right)^{2}\left(1-2 \eta v_{\mathrm{nmo}}^{2}\left(\left(\widehat{\partial_{x} \tau}\right)^{2}+\left(\widehat{\partial_{y} \tau}\right)^{2}\right)\right)=1
\end{aligned}
$$

\section{METHOD: SOLVING THE TILTED ORTHORHOMBIC EIKONAL EQUATION}

Finite-difference solutions of the eikonal equation for anisotropic media with higher order nonlinearity than the isotropic or the elliptically anisotropic eikonal equation give rise to solutions with multiple branches. To suppress nonlinearity in the problem, we apply a fixed-point iteration to a sequence of solutions of a TEA eikonal equation whose source term depends on the traveltimes computed in the previous iteration. We used Aitken's extrapolation method to accelerate the convergence rate of the fixed-point iterations. At every fixed-point iteration, we use the fast-sweeping method to solve the TEA eikonal equation.

\section{Fixed-point iteration}

We rewrite the eikonal equation 6 for TOR media by collecting the TEA portion on the left side

$$
A\left(\widehat{\partial_{x} \tau}\right)^{2}+B\left(\widehat{\partial_{y} \tau}\right)^{2}+C\left(\widehat{\partial_{z} \tau}\right)^{2}=f_{\mathrm{TOR}}(\tau),
$$

where

$$
\begin{gathered}
f_{\mathrm{TOR}}(\tau)=1-D\left(\widehat{\partial_{x} \tau}\right)^{2}\left(\widehat{\partial_{y} \tau}\right)^{2}-E\left(\widehat{\partial_{x} \tau}\right)^{2}\left(\widehat{\partial_{z} \tau}\right)^{2} \\
-F\left(\widehat{\partial_{y} \tau}\right)^{2}\left(\widehat{\partial_{z} \tau}\right)^{2}-G\left(\widehat{\partial_{x} \tau}\right)^{2}\left(\widehat{\partial_{y} \tau}\right)^{2}\left(\widehat{\partial_{z} \tau}\right)^{2}
\end{gathered}
$$

contains the higher order terms. We numerically solve equation 9 using the (implicit) fixed-point iteration

$$
A\left({\widehat{\partial_{x}}}_{n}\right)^{2}+B\left(\widehat{\partial}_{y} \tau_{n}\right)^{2}+C\left(\widehat{\partial}_{z} \tau_{n}\right)^{2}=f_{\mathrm{TOR}}\left(\tau_{n-1}\right),
$$

for $n \geq 1$, where $f_{\mathrm{TOR}}\left(\tau_{0}\right)=1$. For a fixed right side, we solve the TEA eikonal equation 11 using a fast-sweeping method, which will be discussed below. The iterations will converge to the correct solution if the function

$$
g(\tau)=A\left(\widehat{\partial_{x} \tau}\right)^{2}+B\left(\widehat{\partial_{y} \tau}\right)^{2}+C\left(\widehat{\partial_{z} \tau}\right)^{2}-f_{\mathrm{TOR}}(\tau)
$$

is a contraction at each iteration. In other words, for every iteration, $g(\tau)$ has to be Lipschitz continuous (if $g: \mathcal{D} \rightarrow \mathbb{Q}$, then $g$ is Lipschitz continuous on $\mathcal{D}$ with a Lipschitz constant $L$, if $\left|g\left(x_{1}\right)-g\left(x_{2}\right)\right| \leq L\left|x_{1}-x_{2}\right|$ for all $x_{1}, x_{2} \in \mathcal{D}$ ) with a Lipschitz constant less than 1 .

For all cases of practical interest in seismic exploration, and even for a range of parameters well beyond the practical limits, our numerical tests showed that the perturbation term given in equation 10 is sufficiently small, resulting in convergence of the fixed-point iteration.

\section{Aitken's extrapolation}

Assuming the iteration described in equation 11 is convergent, we use Aitken's extrapolation to improve the convergence rate. Given three successive traveltimes obtained by the iteration in equation 11, say $\tau_{n}, \tau_{n+1}$, and $\tau_{n+2}$, Aitken's extrapolation formula for these three terms is given by

$$
A_{n} \approx \tau_{n}-\left(\tau_{n+1}-\tau_{n}\right)^{2}\left(\tau_{n}-2 \tau_{n+1}+\tau_{n+2}\right)^{-1},
$$


resulting in a series $A_{n}$ with a faster convergence rate. One can apply Aitken's extrapolation formula to the sequence obtained in equation 13 to generate a new sequence with a faster convergence rate and repeat the procedure as many times as desired on the consecutive sequences to increase the convergence rate further. For example, given five terms of the fixed-point iteration, $\tau_{1}, \tau_{2}, \tau_{3}, \tau_{4}$, and $\tau_{5}$, let $A_{1}, A_{2}$ and $A_{3}$ be computed by equation 13 . Then, we estimate the traveltime $\tau$ by applying equation 13 to $A_{1}, A_{2}$, and $A_{3}$ :

$$
\tau \approx A_{1}-\left(A_{2}-A_{1}\right)^{2}\left(A_{1}-2 A_{2}+A_{3}\right)^{-1} .
$$

Further details regarding Aitken's extrapolation scheme can be found in Appendix A.

\section{Fast sweeping for a tilted elliptically anisotropic medium}

Our TEA eikonal solver is based on a modification of the fastsweeping method of Zhao (2005). The steps of the algorithm are presented below.

Step 1: Discretization We use the following finite difference approximation for traveltime derivatives:

$$
\begin{aligned}
\widehat{\partial_{x} \tau} \approx & (\cos \phi \cos \psi \cos \theta-\sin \phi \sin \psi)\left(\tau_{i, j, k}-\tau_{x, \min }\right) \Delta x^{-1} s_{x} \\
& +(\cos \phi \sin \psi+\cos \psi \cos \theta \sin \phi)\left(\tau_{i, j, k}-\tau_{y, \min }\right) \Delta y^{-1} s_{y} \\
& +(\cos \psi \sin \theta)\left(\tau_{i, j, k}-\tau_{z, \min }\right) \Delta z^{-1} s_{z}, \\
\hat{\partial_{y}} \tau \approx & (-\cos \psi \sin \phi-\cos \phi \cos \theta \sin \psi)\left(\tau_{i, j, k}-\tau_{x, \min }\right) \Delta x^{-1} s_{x} \\
& +(\cos \phi \cos \psi-\cos \theta \sin \phi \sin \psi)\left(\tau_{i, j, k}-\tau_{y, \min }\right) \Delta y^{-1} s_{y} \\
& -(\sin \psi \sin \theta)\left(\tau_{i, j, k}-\tau_{z, \min }\right) \Delta z^{-1} s_{z}, \\
\hat{\partial_{z}} \tau \approx & -(\cos \phi \sin \theta)\left(\tau_{i, j, k}-\tau_{x, \min }\right) \Delta x^{-1} s_{x}-(\sin \phi \sin \theta) \\
& \times\left(\tau_{i, j, k}-\tau_{y, \min }\right) \Delta y^{-1} s_{y}+\cos \theta\left(\tau_{i, j, k}-\tau_{z, \min }\right) \Delta z^{-1} s_{z}, \\
& i=2,3, \ldots, I-1, j=2,3, \ldots, J-1, k=2,3, \ldots, K-1 .
\end{aligned}
$$

In the above discretization, $\Delta x, \Delta y$, and $\Delta z$ are the grid spacing in the $x$-, $y$-, and $z$-directions, respectively; $\tau_{i, j, k}$ denotes the sought traveltime solution at the grid point $(i, j, k)$;

$$
\begin{aligned}
\tau_{x, \text { min }} & =\min \left(\tau_{i-1, j, k}, \tau_{i+1, j, k}\right), \\
\tau_{y, \text { min }} & =\min \left(\tau_{i, j-1, k}, \tau_{i, j+1, k}\right), \\
\tau_{z, \text { min }} & =\min \left(\tau_{i, j, k-1}, \tau_{i, j, k+1}\right),
\end{aligned}
$$

and

$$
\begin{aligned}
& s_{x}=\left\{\begin{array}{ll}
+1, & \text { if } \tau_{x, \text { min }}=\tau_{i+1, j, k} \\
-1, & \text { if } \tau_{x, \min }=\tau_{i-1, j, k}
\end{array},\right. \\
& s_{y}= \begin{cases}+1, & \text { if } \tau_{y, \text { min }}=\tau_{i, j+1, k} \\
-1, & \text { if } \tau_{y, \min }=\tau_{i, j-1, k}\end{cases} \\
& s_{z}= \begin{cases}+1, & \text { if } \tau_{z, \min }=\tau_{i, j, k+1} \\
-1, & \text { if } \tau_{z, \min }=\tau_{i, j, k-1}\end{cases}
\end{aligned}
$$

In equation 16 , the grid points neighboring the grid node $(i, j, k)$ along the $x$-, $y$ - and $z$-directions are denoted by $\tau_{i \pm 1, j, k}, \tau_{i, j \pm 1, k}$, and $\tau_{i, j, k \pm 1}$, respectively. The sign variables $s_{x}, s_{y}$, and $s_{z}$ ensure that an upwind discretization is used. The only available neighboring grid point, in addition to the appropriate sign variable, is taken for nodes on the boundaries of the computational domain.

Step 2: Initialization We initialize the algorithm by setting the traveltime equal to zero at the source locations and a very large positive value everywhere else.

Step 3: Gauss-Seidel iterations with alternating sweeping order After initialization, an estimate for the solution of the TEA eikonal equation 11 is computed through sweeping (Tsai et al., 2003; Qian et al., 2007). The entire computational domain is swept in the following order repeatedly: (1) $i=1: I, j=1: J, k=1: K$, (2) $i=1: I, j=1: J, k=K: 1$, (3) $i=1: I, j=J: 1, k=1: K$, (4) $i=$ $1: I, j=J: 1, k=K: 1, \quad(5) i=I: 1, j=1: J, k=1: K, \quad(6) i=I: 1$, $j=1: J, k=K: 1, \quad(7) i=I: 1, j=J: 1, k=1: K, \quad(8) i=I: 1, j=J:$ $1, k=K: 1$. For all grid points $(i, j, k)$, we compute an estimated solution, denoted by $\bar{\tau}_{i, j, k}$, to equation 11 from the values of its neighbors $\tau_{i \pm 1, j, k}, \tau_{i, j \pm 1, k}$, and $\tau_{i, j, k \pm 1}$. Then, the traveltime $\tau_{i, j, k}$ is updated to be the smaller one between $\bar{\tau}_{i, j, k}$, and its current value; i.e.,

$$
\tau_{i, j, k}^{\text {new }}=\min \left(\tau_{i, j, k}^{\text {old }}, \bar{\tau}_{i, j, k}\right) .
$$

The estimated solution $\tau_{i, j, k}$ is computed such that it respects the causality criterion.

The causality property of the solution is ensured by checking the monotonicity of the updates (Tsai et al., 2003) along the group velocity directions; i.e., the solution is nondecreasing along the characteristics (see Appendix B for details). After converging to a solution of the TEA eikonal equation, we update the right side of equation 11 with the converged solution and repeat the sweeping for the next fixed-point iteration.

\section{NUMERICAL EXAMPLES}

In this section, we study the behavior of the proposed algorithm. We test our eikonal solver on synthetic TTI and TOR models and compare the eikonal solution with the corresponding first arrival from the wavefield solution. For 2D, we compute the wavefield solution using a finite-difference wavefield extrapolator that is second order in time and fourth order in space. For 3D, we compute the wavefield solution using the algorithm of Song and Alkhalifah (2013).

\section{TILTED AXIS OF SYMMETRY tests}

\section{The 2D homogeneous model}

For TTI tests, we first consider a simple 2D homogeneous TTI model and study the convergence behavior of the algorithm with the increasing number of iterations. The model parameters are $v_{0}=1.8 \mathrm{~km} / \mathrm{s}, \quad v_{\mathrm{nmo}}=2.1 \mathrm{~km} / \mathrm{s}, \quad \eta=0.4$, and $\theta=45^{\circ}$. The source is located at the center of the model $(2.5 \mathrm{~km}, 2.5 \mathrm{~km})$. The grid spacing used is $10 \mathrm{~m}$ in both directions. Figure $1 \mathrm{a}, 1 \mathrm{~b}$, and $1 \mathrm{c}$ shows the wavefield snapshot at $1 \mathrm{~s}$ for a $2 \mathrm{D}$ homogeneous TTI model computed using a finite-difference-based wavefield solver. The corresponding traveltime contour computed using the proposed method is overlaid on the wavefield (in red). Figure 1a, 
$1 \mathrm{~b}$, and $1 \mathrm{c}$ shows traveltime contours after the first, second, and third iterations, respectively. Notice the mismatch between the outer edge of the wavefield and the traveltime contour in Figure 1a. The mismatch reduces significantly after the second iteration, as shown in Figure 1b. Figure 1c shows that the accuracy is further improved after the third iteration. Having computed the first three estimates of traveltimes, we can now use the expression for the Aitken extrapolation, given by equation 13. The traveltime contour using the Aitken extrapolation, shown in Figure 1d, predicts the first-arrival traveltime of the wave visually. a)

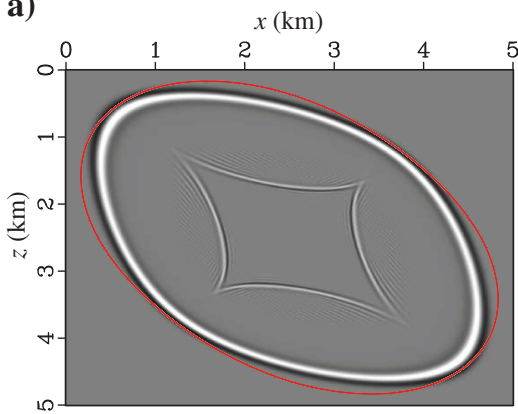

c)

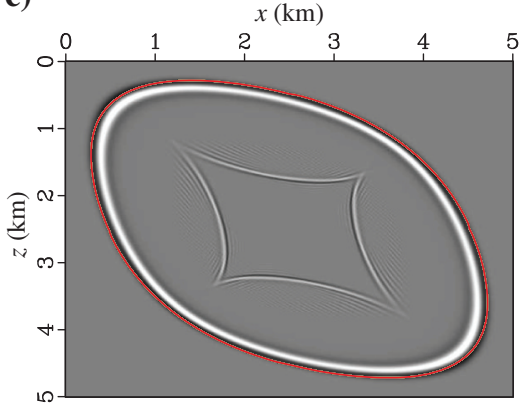

a)

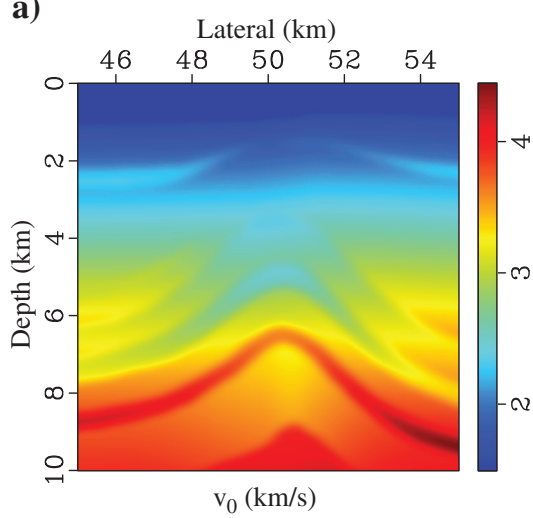

c)

Lateral $(\mathrm{km})$

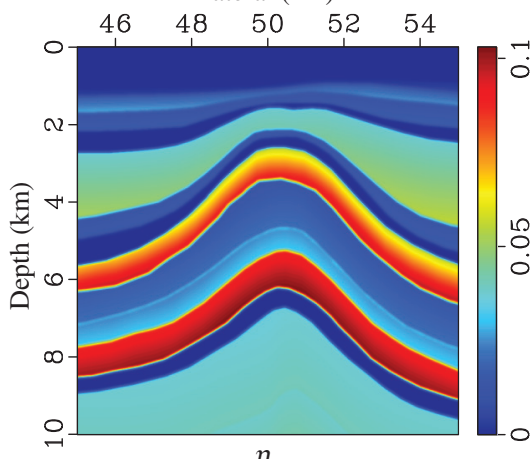

b)

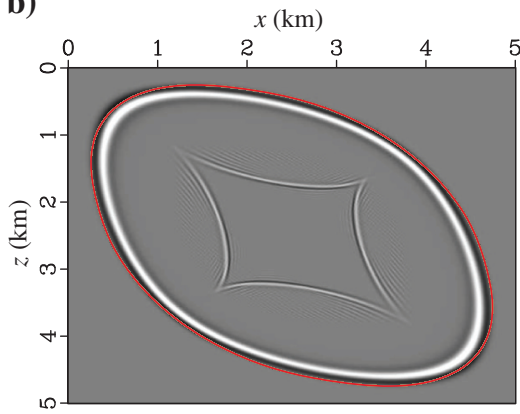

d)

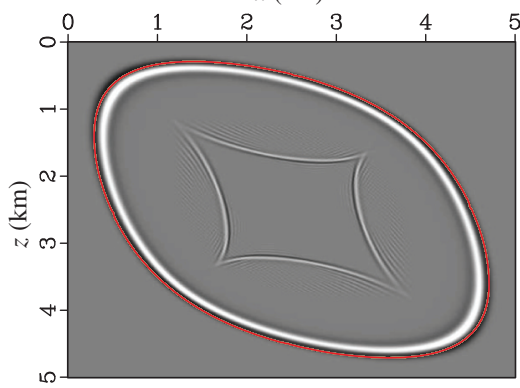

Figure 1. Traveltime contour obtained by the proposed method (in red) overlaid on the wavefield snapshot at $1 \mathrm{~s}$ for the 2D homogeneous TTI model after the (a) first iteration, (b) second iteration, (c) third iteration, and (d) by using Aitken's extrapolation after computing first three traveltime estimates. The star-shaped event in the middle is the S-wave artifact.

b)

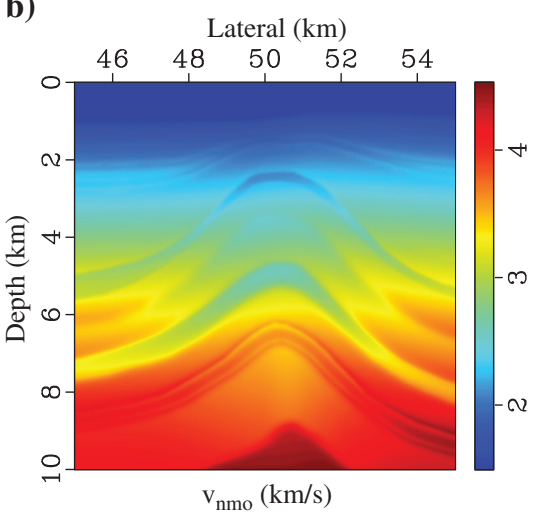

Figure 2. Velocity models (a) $v_{0}$, (b) $v_{\text {nmo }}$, (c) the anellipticity anisotropy parameter $\eta$, and (d) the tilt $\theta$ shown for part of the BP model.

d)

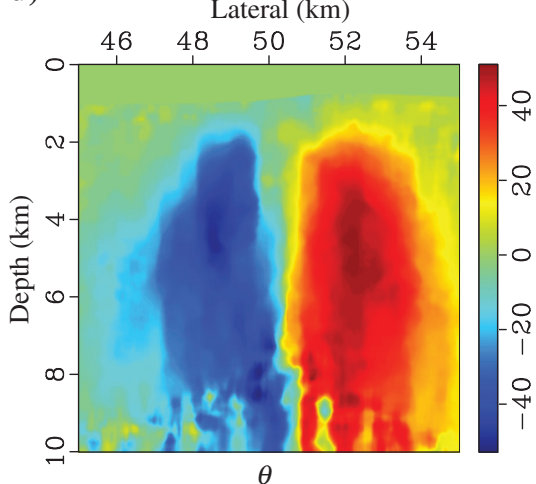




\section{The 2D BP tilted axis of symmetry synthetic model}

Next, we compare the result of our algorithm with the finite-difference-based wavefield extrapolator on parts of the BP TTI model (Shah, 2007).

The portion of the BP TTI model presented in Figure 2 has rapid variations in tilt, which constitutes a challenge. Figure 3 presents the wavefield snapshot for the considered part of the BP model at $1.2 \mathrm{~s}$ emanating from a source at $(50 \mathrm{~km}, 5 \mathrm{~km})$. The corresponding traveltime contour computed using the proposed method is overlaid on the wavefield (in red). The traveltime solution is computed using

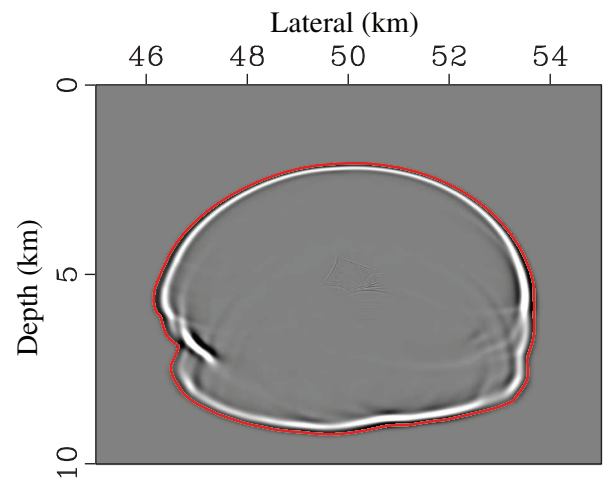

Figure 3. Traveltime contour obtained by the proposed method (in red) overlaid on the wavefield snapshot at $1.2 \mathrm{~s}$ for part of the BP TTI model shown in Figure 2.
Aitken's extrapolation, used after computing the first three traveltime iterations.

The portion of the BP TTI model presented in Figure 4 contains a big salt body. Figure 5 presents the wavefield snapshot for this part of BP model at $1 \mathrm{~s}$ from a source located at $(32 \mathrm{~km}, 5 \mathrm{~km})$. The corresponding traveltime contour computed using the proposed method is overlaid on the wavefield (in red). The traveltime solution is computed using Aitken's extrapolation, used after computing the first three traveltime iterations.

In both cases, the computed traveltime contour matches the first arrival of the wavefield visually.

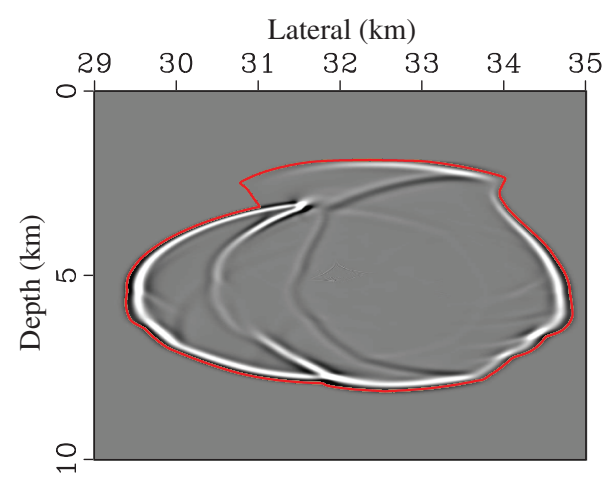

Figure 5. Traveltime contour obtained by the proposed method (in red) overlaid on the wavefield snapshot at $1 \mathrm{~s}$ for part of the BP TTI model shown in Figure 4.
Figure 4. Velocity models (a) $v_{0}$, (b) $v_{\text {nmo }}$, (c) the anellipticity anisotropy parameter $\eta$ (c), and (d) the tilt $\theta$ shown for part of the BP model. a)

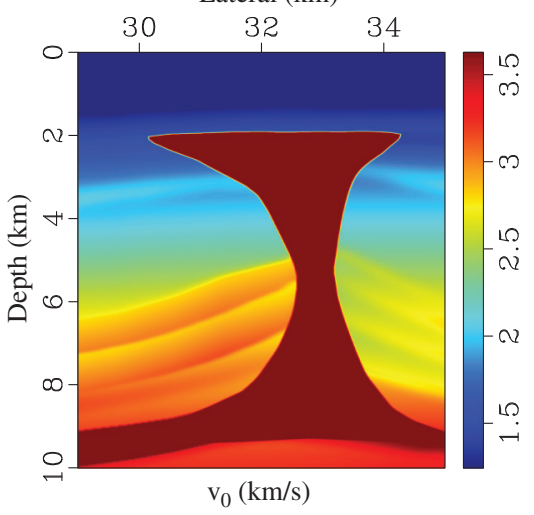

c)

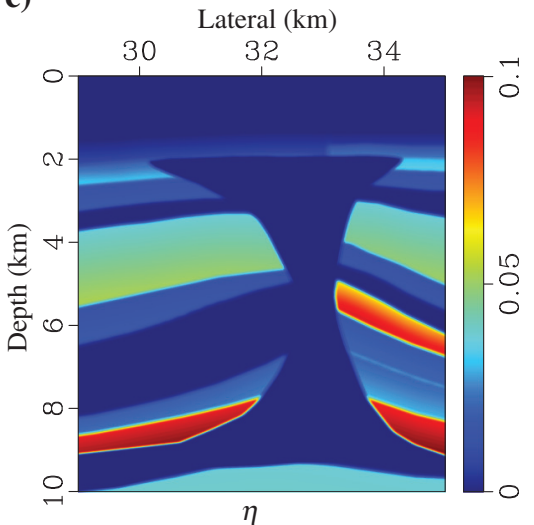

b)

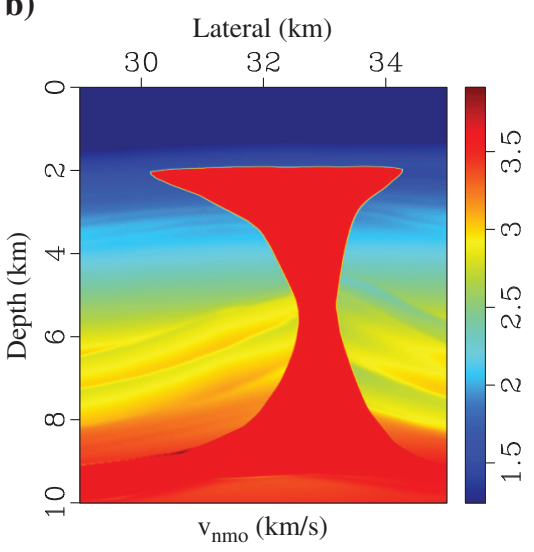

d)

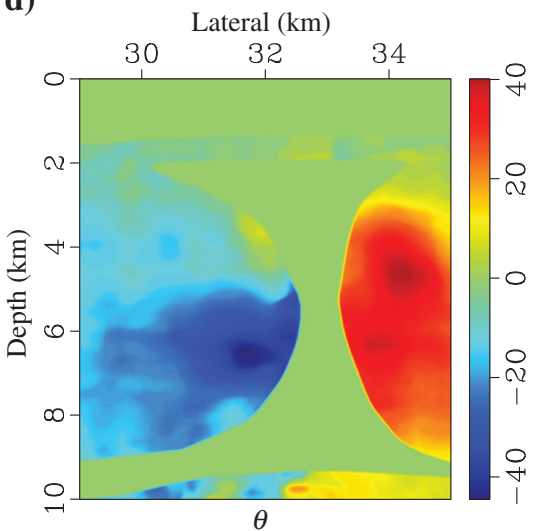




\section{The 3D homogeneous model}

For 3D, we consider a homogeneous TTI model with $v_{0}=$ $2 \mathrm{~km} / \mathrm{s}, v_{\text {nmo }}=2.2 \mathrm{~km} / \mathrm{s}, \eta=0.2, \theta=\phi=45^{\circ}$, and $\psi=0^{\circ}$. Figure $6 \mathrm{a}, 6 \mathrm{~b}$, and $6 \mathrm{c}$ shows wavefield snapshots at $0.5 \mathrm{~s}$ for the inline, crossline, and depth slices respectively, for a source located at (1.5, $1.5,1.5 \mathrm{~km}$ ). The wavefield extrapolation algorithm is based on the low-rank approximation (Song and Alkhalifah, 2013). We also plot traveltime contours at $0.5 \mathrm{~s}$ obtained using the eikonal solver, overlaid on the wavefield (in red). The traveltime contour obtained matches the first arrival of the wavefield visually.

\section{Tilted orthorhombic test model}

\section{The 3D homogeneous model}

For TOR tests, first we consider a homogeneous TOR model with $v_{0}=2 \mathrm{~km} / \mathrm{s}, \quad v_{\text {nmo1 }}=2.2 \mathrm{~km} / \mathrm{s}, \quad v_{\text {nmo2 }}=2.5 \mathrm{~km} / \mathrm{s}, \quad \eta_{1}=0.2$, $\eta_{2}=0.3, \theta=\phi=45^{\circ}, \psi=0^{\circ}$, and $\gamma=1$. Figure $7 \mathrm{a}, 7 \mathrm{~b}$, and $7 \mathrm{c}$ shows wavefield snapshots at 0.5 second for the inline, crossline, and depth slices, respectively, for a source located at $(1.5 \mathrm{~km}$, $1.5 \mathrm{~km}, 1.5 \mathrm{~km})$. The corresponding traveltime contours obtained using the eikonal solver are overlaid on the wavefield (in red). The traveltime contour obtained using the eikonal solver matches the first arrival of the wavefield visually. For this case, we repeated Aitken's extrapolation twice on the first five traveltime estimates of the fixed-point iteration, to approximate the solution of equation 9 .

\section{The $3 D$ layered synthetic model}

Finally, we consider a three-layer TOR model. Each layer is flat and has a dimension of $1 \mathrm{~km} \times 1 \mathrm{~km} \times 1 \mathrm{~km}$. Medium parameters for the three-layer model are as follows: layer $1: v_{0}=1.5 \mathrm{~km} / \mathrm{s}$, $v_{1}=1.8 \mathrm{~km} / \mathrm{s}, v_{2}=1.9 \mathrm{~km} / \mathrm{s}, \eta_{1}=0.1, \eta_{2}=0.15, \theta=20^{\circ}, \phi=20^{\circ}$; layer $2: v_{0}=1.8 \mathrm{~km} / \mathrm{s}, v_{1}=2.05 \mathrm{~km} / \mathrm{s}, v_{2}=2.15 \mathrm{~km} / \mathrm{s}, \eta_{1}=0.15$, $\eta_{2}=0.2, \theta=30^{\circ}, \phi=10^{\circ}$; and layer $3: v_{0}=2.0 \mathrm{~km} / \mathrm{s}, v_{1}=2.3 \mathrm{~km} / \mathrm{s}$, $v_{2}=2.4 \mathrm{~km} / \mathrm{s}, \eta_{1}=0.2, \eta_{2}=0.3, \theta=40^{\circ}, \phi=-20^{\circ}$. We use layers 1 , 2 , and 3 to refer to the top, middle, and bottom layers, respectively. a)

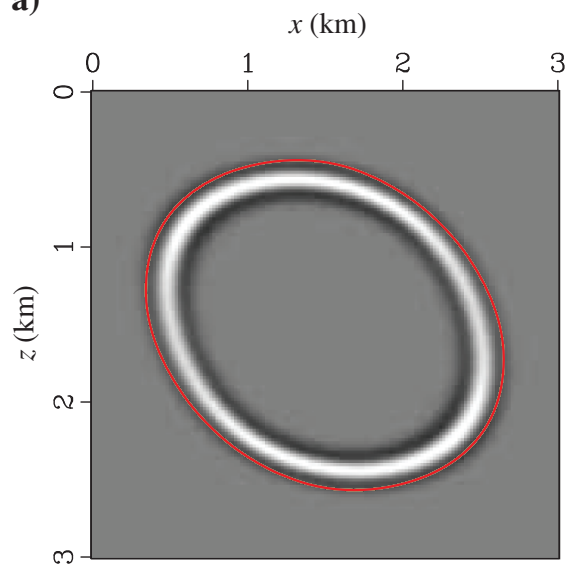

b)

$$
3
$$

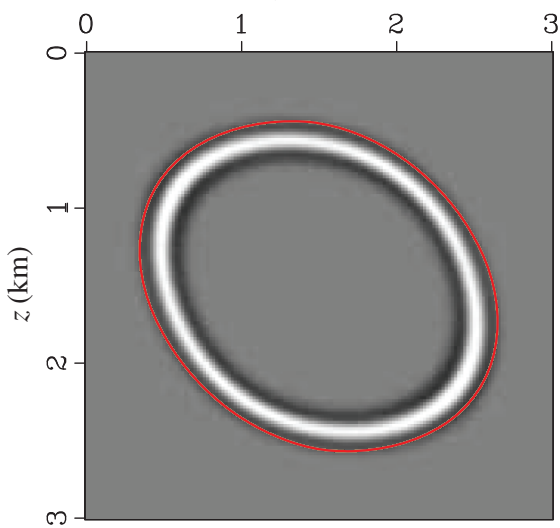

c)

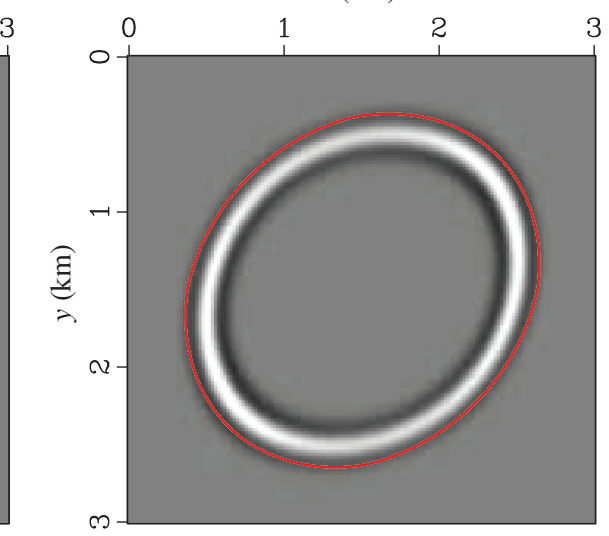

Figure 6. Traveltime contours obtained by the proposed method (in red) overlaid on the wavefield snapshots at $0.5 \mathrm{~s}$ for the $3 \mathrm{D}$ homogeneous TTI model. (a) The inline slice at $y=1.5 \mathrm{~km}$, (b) the crossline slice at $x=1.5 \mathrm{~km}$, and (c) the depth slice at $z=1.5 \mathrm{~km}$.

a)

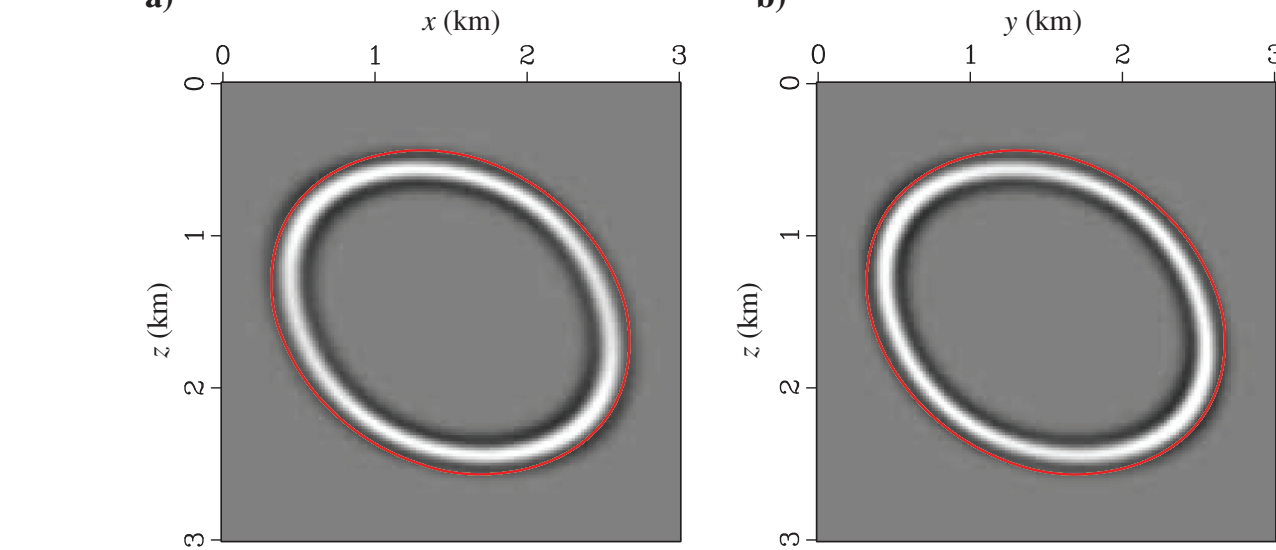

b)

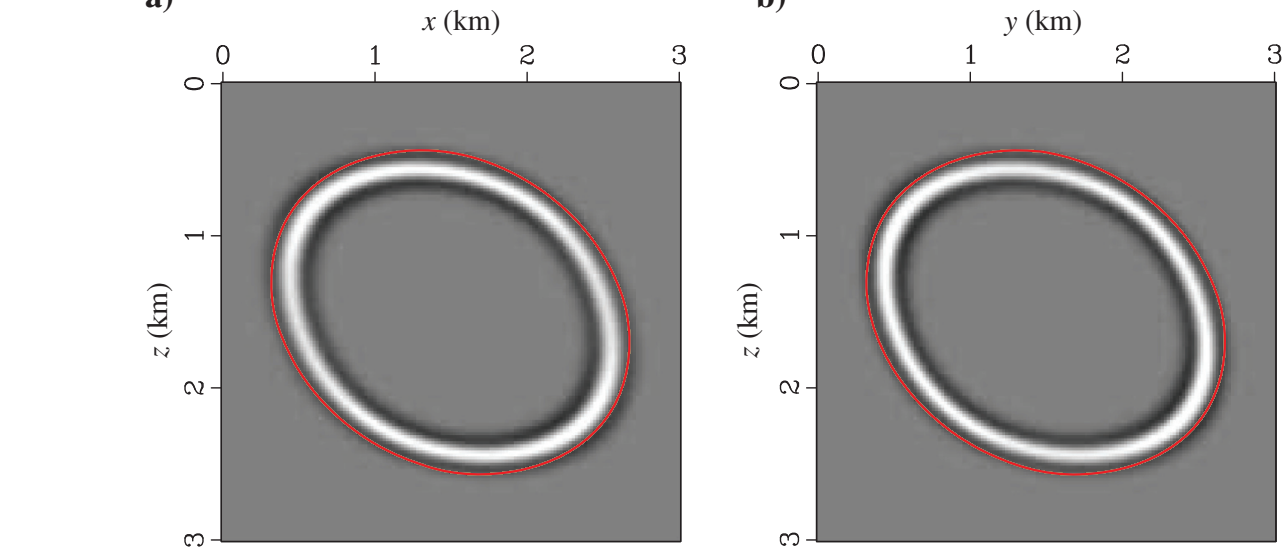

c)

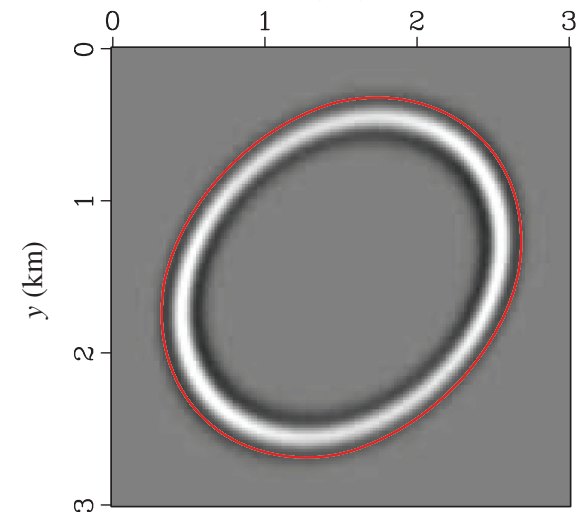

Figure 7. Traveltime contours obtained by the proposed method (in red) overlaid on the wavefield snapshots at $0.5 \mathrm{~s}$ for a homogeneous TOR model. (a) The inline slice at $y=1.5 \mathrm{~km}$, (b) the crossline slice at $x=1.5 \mathrm{~km}$, and (c) the depth slice at $z=1.5 \mathrm{~km}$. 
a)

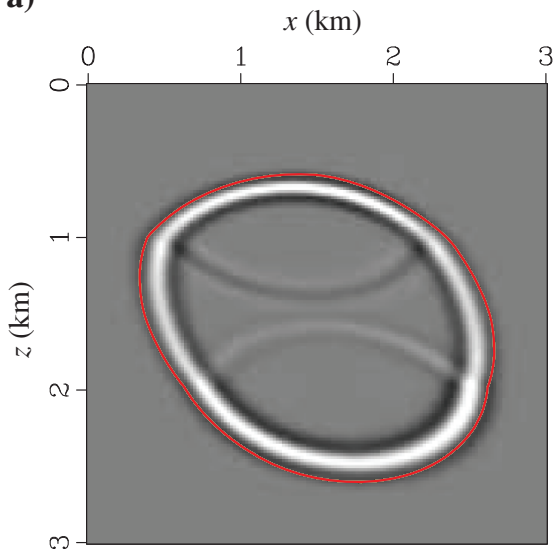

b)

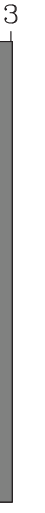

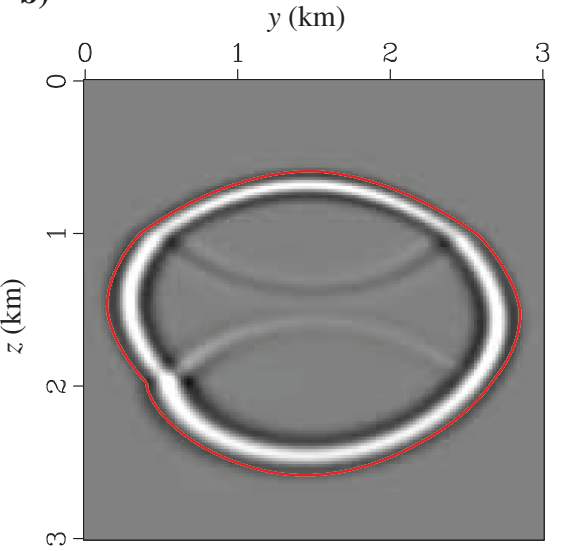

c)

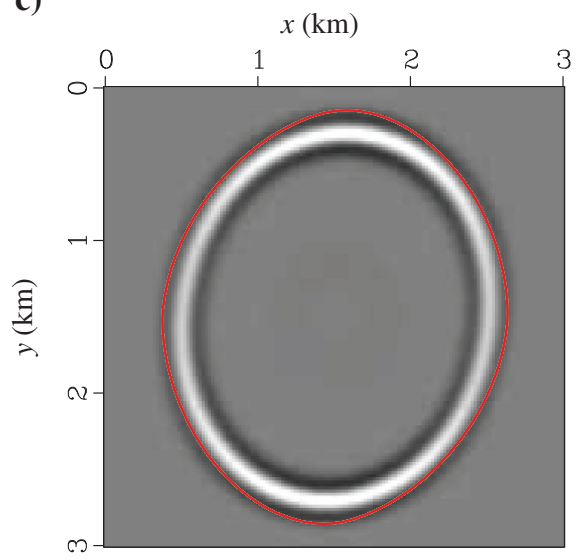

Figure 8. Traveltime contours obtained by the proposed method (in red) overlaid on the wavefield snapshots at $0.5 \mathrm{~s}$ for a three-layered TOR model. (a) The inline slice at $y=1.5 \mathrm{~km}$, (b) the crossline slice at $x=1.5 \mathrm{~km}$, and (c) the depth slice at $z=1.5 \mathrm{~km}$.

Constant values of $\gamma=1$ and $\psi=0^{\circ}$ are used in the model. Figure $8 \mathrm{a}, 8 \mathrm{~b}$, and $8 \mathrm{c}$ shows wavefield snapshots at $0.5 \mathrm{~s}$ for inline, crossline and depth slices, respectively, emanating from a source at $(1.5 \mathrm{~km}, 1.5 \mathrm{~km}, 1.5 \mathrm{~km})$. The corresponding traveltime contour computed using the proposed method is overlaid on the wavefield (in red). The computed traveltime contour matches the first arrival of the wavefield visually. For this case, we repeated Aitken's extrapolation twice on the first five traveltime estimates of the fixed-point iteration, to approximate the solution of equation 9.

\section{CONCLUSION}

Computing accurate first-arrival traveltimes of the quasi-P-waves in anisotropic media is important for high-end near-surface modeling, microseismic-source localization, and fractured-reservoir characterization, and it requires solving an anisotropic eikonal equation. Anisotropy deviating from elliptical anisotropy introduces higher order nonlinearity into the eikonal equation, which makes solving the eikonal equation a challenge. We address this challenge by using the solution of tilted elliptical anisotropy to build an iterative method for solving the TTI and TOR eikonal equations. At each iteration, we updated the source term in the TEA eikonal solver that encompasses most of the nonlinear effects of the higher order anisotropic eikonal equation. To speed up the convergence rate of the iterative algorithm, we used Aitken's extrapolation.

Our numerical tests show that the proposed algorithm is feasible and accurate, even for strongly anisotropic and complex structures. Furthermore, our approach can be easily extended to anisotropic media with lower symmetries, such as the monoclinic or even the triclinic media, by considering the additional higher order terms for these media as source function. Adaption of the proposed algorithm to compute first-arrival traveltimes for $\mathrm{qS}$-waves remains a topic of further investigation.

\section{ACKNOWLEDGMENTS}

We would like to thank Schlumberger for financial support and permission to publish the results. We are also grateful to K. Osypov, T. Alkhalifah, and I. Pšencík for useful discussions. We greatly appreciate the reviewers for many valuable suggestions that helped considerably in improving the quality of the paper. We also extend thanks to BP for releasing the benchmark synthetic model. Finally, we thank all the contributors for making Madagascar a viable opensource research-software project.

\section{APPENDIX A}

\section{AITKEN'S EXTRAPOLATION METHOD}

Aitken's extrapolation method and its generalizations due to Lubkin are a central component of the mathematical theory of sequence extrapolation. Formally stated, Aitken's extrapolation method is a nonlinear sequence transformation. Given a sequence $\left\{x_{n}\right\} \in \mathbb{C}$, Aitken's extrapolation method is defined as

$$
A_{n}=\frac{x_{n} x_{n+2}-x_{n+1}^{2}}{x_{n}-2 x_{n+1}+x_{n+2}} .
$$

The computationally stable form of this transformation is given by

$$
A_{n}=x_{n}-\frac{\left(\Delta x_{n}\right)^{2}}{\Delta^{2} x_{n}}=x_{n+1}-\frac{\left(\Delta x_{n}\right)\left(\Delta x_{n+1}\right)}{\Delta^{2} x_{n}} .
$$

Under certain conditions on the convergence of the sequence $x_{n}$, the transformed sequence $A_{n}$ converges to the same limit as $\left\{x_{n}\right\}$, only at a much faster rate. This result has proven to be particularly useful for accelerating the convergence rate of nonlinear fixed-point iterations. Let us define the fixed-point iteration

$$
x_{n+1}=g\left(x_{n}\right)
$$

for some nonlinear function $g$, which is assumed to be infinitely differentiable in the neighborhood of a fixed-point $\hat{x}$. Furthermore, assume that in this neighborhood of $\hat{x}, g$ satisfies $\left|g^{\prime}(x)\right|<1$. Under these assumptions, the fixed-point iteration will converge, though possibly very slowly, to the fixed-point $\hat{x}$. Moreover, one can show that the iterates $x_{n}$ in the fixed-point iteration can be related to $\hat{x}$ asymptotically as

$$
x_{n} \sim \hat{x}+\sum_{k=0}^{\infty} \alpha_{k}\left(g^{\prime}(\hat{x})\right)^{k n}, \quad n \rightarrow \infty ;
$$


see Sidi (2003) for further details related to this result and the results that follow.

One can then show that the Aitken's extrapolation of the sequence $\left\{x_{n}\right\}$ is related asymptotically to the fixed point via

$$
A_{n} \sim \hat{x}+\sum_{k=0}^{\infty} \beta_{k}\left(g^{\prime}(\hat{x})\right)^{(k+1) n}, \quad n \rightarrow \infty .
$$

Now let $A_{n}^{(j)}$ be the sequence obtained by iteratively applying the Aitken transformation $j$ times. Then, by iteratively applying the Aitken transformation to the original sequence $\left\{x_{n}\right\}$, one can obtain the following accelerated convergence result (see Sidi [2003] for further details):

$$
A^{(j)}\left(g\left(x_{n}\right)\right)-\hat{x}=\mathcal{O}\left(\left|g^{\prime}(\hat{x})\right|^{(n+1) j}\right), \quad j \rightarrow \infty .
$$

Hence, for our fixed-point iteration, at each point in the spatial discretization we may accelerate the convergence of the fixed-point iterations in the traveltime computation via the Aitken's extrapolation method.

\section{APPENDIX B}

\section{CAUSALITY CRITERIA}

To ensure causality, the update sequence must be monotone. This means that the updated traveltime value of a grid node must be greater than or equal to the neighboring grid nodes that are used to form the finite difference stencil. This amounts to the condition (Tsai et al., 2003)

$$
\frac{\partial \tau}{\partial x} \cdot \frac{\partial H}{\partial p_{x}}+\frac{\partial \tau}{\partial y} \cdot \frac{\partial H}{\partial p_{y}}+\frac{\partial \tau}{\partial z} \cdot \frac{\partial H}{\partial p_{z}} \geq 0,
$$

where $H\left(x, y, z, p_{x}, p_{y}, p_{z}\right)$ represents the Hamiltonian and $p_{x}, p_{y}$ and $p_{z}$ denote the slowness vectors in the $x$-, $y$-, and $z$-directions, respectively. The above condition, in equation B-1 ensures that the solution is nondecreasing along the characteristics. However, in the case of using the one-sided finite-difference approximation, as we do here, the condition in equation B-1 is equivalent to demanding that the partial derivatives of traveltimes $\left(\frac{\partial \tau}{\partial x}, \frac{\partial \tau}{\partial y}, \frac{\partial \tau}{\partial z}\right)$ and their corresponding components of the characteristics directions $\left(\frac{\partial H}{\partial p_{x}}, \frac{\partial H}{\partial p_{y}}, \frac{\partial H}{\partial p_{z}}\right)$ have the same sign; i.e.,

$$
\frac{\partial \tau}{\partial x} \cdot \frac{\partial H}{\partial p_{x}} \geq 0, \quad \frac{\partial \tau}{\partial y} \cdot \frac{\partial H}{\partial p_{y}} \geq 0, \quad \frac{\partial \tau}{\partial z} \cdot \frac{\partial H}{\partial p_{z}} \geq 0
$$

The fast-sweeping method developed by Tsai et al. (2003) is valid only for convex Hamiltonians; however, ensuring that the updated traveltimes honor the criterion in equation B-1 means that our methodology is not limited only to convex Hamiltonians.

\section{REFERENCES}

Alkhalifah, T., 1998, Acoustic approximations for processing in transversely isotropic media: Geophysics, 63, 623-631, doi: 10.1190/1.1444361.

Alkhalifah, T., 2003, An acoustic wave equation for orthorhombic anisotropy: Geophysics, 68, 1169-1172, doi: 10.1190/1.1598109.

Alkhalifah, T., and S. Fomel, 2011, The basic components of residual migration in VTI media using anisotropy continuation: Journal of Petroleum
Exploration and Production Technology, 1, 17-22, doi: 10.1007/s13202011-0006-6.

Al-Shuhail, A., S. I. Kaka, and M. Jervis, 2013, Enhancement of passive microseismic events using seismic interferometry: Seismological Research Letters, 84, 781-784, doi: 10.1785/0220130012.

Atkinson, K., 1989, An introduction to numerical analysis: John Wiley \& Sons.

Bakulin, A., V. Grechka, and I. Tsvankin, 2000, Estimation of fracture parameters from reflection seismic data-part I: HTI model due to a single fracture set: Geophysics, 65, 1788-1802, doi: 10.1190/1.1444863.

Baofu, C., X. Dingyu, R. Xiaoqiao, and C. Chunhuaet al., 2008, The firstarrival tomographic inversion and its application to identify thick nearsurface structures: 78th Annual International Meeting, SEG, Expanded Abstracts, 3255-3259.

Bevc, D., 1997, Imaging complex structures with semi-recursive Kirchhoff migration: Geophysics, 62, 577-588, doi: 10.1190/1.1444167.

Cěrvený, V., 2001, Seismic ray theory: Cambridge University Press.

Cristiani, E., 2009, A fast marching method for Hamilton-Jacobi equations modeling monotone front propagations: Journal of Scientific Computing, 39, 189-205, doi: 10.1007/s10915-008-9257-x.

Dellinger, J., and W. Symes, 1997, Anisotropic finite-difference traveltimes using a Hamilton-Jacobi solver: 67th Annual International Meeting, SEG, Expanded Abstracts, 1786-1789.

Dellinger, J., and J. van Trier, 1990, Anisotropic finite-difference traveltimes: Stanford Exploration Project, SEP-65.

Konukoglu, E., M. Sermesant, O. Clatz, J.-M. Peyrat, H. Delingette, and N. Ayache, 2007, A recursive anisotropic fast marching approach to reaction diffusion equation: Application to tumor growth modeling: Information Processing in Medical Imaging, 4584, 687-699, doi: 10.1007/978-3540-73273-0_57.

Ma, X, and T. Alkhalifah, 2013, qP wave traveltime computation by an iterative approach: 75th Annual International Conference and Exhibition, EAGE, Extended Abstracts, WE0209.

Osher, S., 1993, A level set formulation for the solution of the Dirichlet problem for Hamilton-Jacobi equations: SIAM Journal on Mathematical Analysis, 24, 1145-1152, doi: 10.1137/0524066.

Qian, J., and W. W. Symes, 2001, Paraxial eikonal solvers for anisotropic quasi-P travel times: Journal of Computational Physics, 173, 256-278, doi: 10.1006/jcph.2001.6875.

Qian, J., Y.-T. Zhang, and H.-K. Zhao, 2007, A fast sweeping method for static convex Hamilton-Jacobi equations: Journal of Scientific Computing, 31, 237-271, doi: 10.1007/s10915-006-9124-6.

Rouy, E., and A. Tourin, 1992, A viscosity solutions approach to shapefrom-shading: SIAM Journal on Numerical Analysis, 29, 867-884, doi: $10.1137 / 0729053$

Sethian, J. A., and A. M. Popovici, 1999, 3-D traveltime computation using the fast marching method: Geophysics, 64, 516-523, doi: 10 $.1190 / 1.1444558$.

Sethian, J. A., and A. Vladimirsky, 2003, Ordered upwind methods for static Hamilton-Jacobi equations: Theory and algorithms: SIAM Journal on Numerical Analysis, 41, 325-363, doi: 10.1137/S0036142901392742.

Shah, H., 2007, The 2007 BP anisotropic velocity-analysis benchmark: 69th Annual International Conference and Exhibition, EAGE, workshop.

Sidi, A., 2003, Practical extrapolation methods: Theory and applications: Cambridge University Press.

Song, X., and T. Alkhalifah, 2013, Modeling of pseudoacoustic P-waves in orthorhombic media with a low-rank approximation: Geophysics, 78, no. 4, C33-C40, doi: 10.1190/geo2012-0144.1.

Stoep, D. V., 1966, Velocity anisotropy measurements in wells: Geophysics, 31, 900-916, doi: 10.1190/1.1439822.

Stovas, A., and T. Alkhalifah, 2012, A new traveltime approximation for TI media: Geophysics, 77, no. 4, C37-C42, doi: 10.1190/geo2011-0158.1.

Taillandier, C., M. Noble, H. Chauris, and H. Calandra, 2009, First-arrival traveltime tomography based on the adjoint-state method: Geophysics, 74, no. 6, WCB1-WCB10, doi: 10.1190/1.3250266.

Tsai, Y.-H. R., L.-T. Cheng, S. Osher, and H.-K. Zhao, 2003, Fast sweeping algorithms for a class of Hamilton-Jacobi equations: SIAM Journal on Numerical Analysis, 41, 673-694, doi: 10.1137/S0036142901396533.

Tsvankin, I., J. Gaiser, V. Grechka, M. van der Baan, and L. Thomsen, 2010, Seismic anisotropy in exploration and reservoir characterization: An overview: Geophysics, 75, no. xx, 75A15-75A29, doi: 10.1190/1.3481775.

van Trier, J., and W. W. Symes, 1991, Upwind finite-difference calculation of traveltimes: Geophysics, 56, 812-821, doi: 10.1190/1.1443099.

Vidale, J. E., 1990, Finite-difference calculation of traveltimes in three dimensions: Geophysics, 55, 521-526, doi: 10.1190/1.1442863.

Waheed, U., and T. Alkhalifah, 2013, Efficient traveltime solutions of the TI acoustic eikonal equation: 75th Annual International Conference and Exhibition, EAGE, Extended Abstracts, Th0611.

Waheed, U., T. Alkhalifah, and H. Wang, 2015, Efficient traveltime solutions of the acoustic TI eikonal equation: Journal of Computational Physics, 282, 62-76, doi: 10.1016/j.jcp.2014.11.006.

Waheed, U., I. Pšenčík, V. Cervený, E. Iversen, and T. Alkhalifah, 2013, Two-point paraxial travel time formula for inhomogeneous isotropic 
Waheed et al.

and anisotropic media: Tests of accuracy: Geophysics, 78, no 5, WC65WC80, doi: 10.1190/geo2012-0406.1.

Waheed, U., C. E. Yarman, and G. Flagg, 2014, An iterative fast sweeping based eikonal solver for tilted orthorhombic media: 84th Annual International Meeting, SEG, Expanded Abstracts, 480-485.
Zhang, Y.-T., H.-K. Zhao, and J. Qian, 2006, High order fast sweeping methods for static Hamilton-Jacobi equations: Journal of Scientific Computing, 29, 25-56, doi: 10.1007/s10915-005-9014-3.

Zhao, H., 2005, A fast sweeping method for eikonal equations: Mathematics of Computation, 74, 603-628, doi: 10.1090/S0025-5718-04-01678-3. 